\title{
Bessere Rahmenbedingungen allein beseitigen Armut nicht! Eine theoriegeleitete Vier Ebenen-Strategie für entwicklungspolitische Interventionen
}

\section{Theo Rauch, Bonn}

\section{Entwicklungspolitik zwischen Baobab und Washington}

Was ist das Handwerkszeug der Zunft der Entwicklungshelfer? Darüber hat man sich in der entwicklungspolitischen Branche stets gestritten. Einigkeit darüber besteht heute weniger denn je, wurde vielleicht auch nie angestrebt. In der Tat tummeln sich vielerlei Gewerbe mit ihren Instrumenten auf dem weiten Feld der Entwicklungszusammenarbeit (EZ). Die Techniker, die Agraringenieure, Tierärzte, Schreinermeister, Hoch-, Tief- und Wasserbauingenieure, auch die Ärzte und Lehrer, welche das Feld vor drei Jahrzehnten beherrscht hatten, befinden sich eindeutig in der Defensive. «Das können die Partnerländer heute selbst» heisst es. Die Vertreter und Vertreterinnen der betreffenden Berufsgruppen haben sich umschulen lassen oder aber sie arbeiten dort, wo die Partnerländer selbst bezahlen und selbst bestimmen, wen sie brauchen: In reichen Erdölländern, wie Saudi-Arabien. Die Manager, auch sie gelten als «out». Wahrscheinlich nicht deshalb, weil sie sich überflüssig gemacht haben, sondern im Gegenteil, weil sie der Versuchung erlagen, den Fehltritt der «Ersatzvornahme» zu begehen, d.h. die Region, den Verband, die Genossenschaft, in welcher sie bessere Managementverfahren einführen sollten, einfachheitshalber gleich selbst in die Hand zu nehmen.

In den 90er-Jahren hatte das Handwerkszeug der Pädagogen, Psychologen und Kommunikationsfachleute Konjunktur: «Entwicklung setzt beim Menschen an», war das Credo. Befähigung, Motivation, Animation, Mobilisierung heissen die Instrumentenkästen, mit denen die Fachleute der Entwicklungsorganisationen und die von ihnen angelernten, einheimischen Kolleginnen und Kollegen in die Dörfer gingen. Gleichzeitig boten die Soziologen, Ethnologen, Geographen, Landschafts- und Stadtplaner, Verwaltungswissenschaftler ihre diversen Analysekompetenzen an: Zielgruppenanalyse, Gender-Analyse, Geographische Informationssysteme, Regionalanalyse, Landeignungsanalyse, Organisationsdiagnose wurden in den Instrumentenköfferchen des Gutachtergewerbes mal einzeln, mal im Kombi-Pack feilgeboten.

All das kennzeichnet auch heute noch den «Bauchladen» der Entwicklungszusammenarbeit. Der Mainstream aber bewegt sich zu den höheren Etagen hin. «Makro» ist «in». Die Einschätzung, dass ohne eine Veränderung der Rahmenbedingungen alle Bemühungen auf lokaler und regionaler Ebene nicht zu dauerhafter und breitenwirksamer Entwicklung beitragen können, sondern bestenfalls zu künstlichen, temporären Insellösungen führen würden, hat sich mittlerweile auf breiter Ebene durchgesetzt. Bereits Ende der 80er-Jahre votierten die WeLtBank und der INTERNATIONALE WäHRUNGSFONDS (IWF) für eine Abkehr von lokalen und regionalen Fördermaßnahmen durch internationale, staatliche Hilfsorganisationen und für eine Konzentration auf die Veränderung der nationalen wirtschaftspolitischen und institutionellen Rahmenbedingungen durch eine gebündelte Unterstützung von Strukturanpassungsund Sektorinvestitionsprogrammen (SAP und SIP). Anfang der 90er-Jahre gewannen Sozial- und Politikwissenschaftler und -wissenschaftlerinnen mehr Einfluss und erweiterten das Spektrum der zu verändernden Rahmenbedingungen auf den gesellschaftspolitischen Rahmen: Demokratisierung, good governance und die Förderung der Zivilgesellschaft wurden zentrale Handlungsfelder der Entwicklungszusammenarbeit.

Im Zusammenhang mit der Globalisierungsdebatte und dem zunehmenden Einfluss sog. linker, entwicklungshilfeskeptischer Strömungen in westeuropäischen Ländern, wurde das Spektrum der zu verändernden Rahmenbedingungen um die globale Ebene erweitert. So versteht beispielsweise das deutsche Entwicklungshilfeministerium (Bundesministerium für wirtschaftliche Zusammenarbeit und Entwicklung, BMZ) seit der Machtübernahme durch die rot-grüne Regierung die Entwicklungszusammenarbeit als globale Strukturpolitik. Neben dem klassischen Bereich der Förderung von Projekten und Programmen im Rahmen der bilateralen, europäischen und multilateralen Entwicklungszusammenarbeit gehören die «Mitgestaltung internationaler Regelwerke und Konzepte» (im Rahmen der WTO und von UN-, insbesondere ILO-Konferenzen) und die Inlandarbeit (z.B. Informationsarbeit in den Bereichen Menschenrechte, Klimaschutz, Kinderarbeit oder fair trade) zu den zentralen Handlungsfeldern der Entwicklungspolitik. Programme und Projekte in den Partnerländern sollen primär daran orientiert sein, bei der Umsetzung von internationalen Vereinbarungen zu helfen (vgl. WiecZoreK-Zeul 2000). Hierbei spielt die Unterstützung von «Makrostrukturreformen» durch Regierungsberater und -beraterinnen eine zentrale Rolle. 
Die Frage, auf welcher Ebene die Entwicklungszusammenarbeit am wirkungsvollsten intervenieren könnte, hat also im Laufe der Jahrzehnte unterschiedliche Antworten erfahren. Selten aber war sie Gegenstand einer systematischen vergleichenden Erörterung oder Analyse. Meist war sie das Feld einer Auseinandersetzung einander konkurrierender Fachrichtungen nach dem Motto: «Diese Fehlschläge sind die Folge einer sträflichen Vernachlässigung der Fachkenntnisse der xy-Disziplin». Verschiebungen der Interventionsebenen und -felder vollzogen sich als Pendelbewegungen im Sinne einer Reaktion auf das (tatsächliche oder vermeintliche) Scheitern des einen oder anderen Ansatzes. Auch fachübergreifende Paradigmenwechsel in den Wissenschaften, etwa die postmoderne Tendenz weg vom Strukturalismus hin zu akteursorientierten Ansätzen mag die vorherrschenden Antworten auf die (nicht explizit gestellte) Frage nach der effektivsten Interventionsebene beeinflusst haben.

Die Veränderungen in der entwicklungspolitischen Praxis waren meist weniger dramatisch als jene in den Konzeptionspapieren, was einem gewissen personellen und organisatorischen Beharrungsvermögen zuzuschreiben ist. Dieses Beharrungsvermögen fand seine Legitimation durch einen breiten organisationspraktischen Grundkonsens, dass alle Beteiligten wichtig seien und man komplementär auf unterschiedlichen Ebenen intervenieren müsse.

\section{Zur Notwendigkeit systematisch reflektierter Entscheidungen über Interventionen}

Wären Eingriffe stets harmlos, bräuchte man sich über den angebotsorientierten Interventionspragmatismus der Entwicklungszusammenarbeit keine Gedanken zu machen. Aber schon die Alltagserfahrung zeigt: Interventionen können stören, sie können uns von nützlichem Tun abhalten, unsere Energien unnötig absorbieren, Schaden anrichten. Hier sollen nicht die alten und fortwährenden Auseinandersetzungen zwischen liberalen und sozialistischen Staats- und Wirtschaftsphilosophien bezüglich der Schädlichkeit und Notwendigkeit staatlicher Interventionen in gesellschaftliches und wirtschaftliches Handeln wiederholt werden. Was aber in dieser Debatte auch jene gelernt haben, die den Selbstregulierungskräften des freien Spiels der Kräfte misstrauen, und die die Notwendigkeit einer gestaltenden Rolle staatlicher Politik zur sozialen und ökologischen Regulierung ökonomischer Prozesse sehen, ist, dass es sich bei staatlicher Politik um sensible Eingriffe in komplexe Systeme handelt, die, wenn sie unreflektiert erfolgen, Gefahr laufen

- Selbstregulierungskräfte zu unterminieren,

- ihre Ziele aufgrund vernachlässigter Wirkungsfaktoren nicht zu erreichen,
- unerwünschte Nebenwirkungen zu erzeugen und so in eine fortwährende «Flickschusterei» zu münden.

Besonders prekär sind entwicklungspolitische Interventionen, da es sich um die Einmischung in Angelegenheiten anderer Länder (Legitimationsproblem) und in oft wenig bekannte kulturspezifische Systemzusammenhänge handelt (Kompetenzproblem). Wer daraus nun die Forderung nach einer entwicklungspolitischen Abstinenz ableitet, dem ist entgegenzuhalten, dass jegliche Form von Unterstützung eine Intervention mit all ihrer Problematik impliziert. Auch der Transfer unkonditionierter Geldmittel beeinflusst die gesellschaftliche Verteilung knapper Ressourcen und damit von Macht, z.B. zwischen staatlichen und zivilgesellschaftlichen Akteuren, zwischen Zentralstaat und regionalen bzw. lokalen Gebietskörperschaften. Und selbst die völlige Abschaffung entwicklungspolitisch begründeter Interventionen würde das Feld der Intervention in einer sich globalisierenden Weltwirtschaft und -politik nur anderen Akteuren und deren Zielen und Interessen überlassen.

Wenn also eine entwicklungspolitische Abstinenz keine realistische Option ist, muss es darum gehen, einem angebotsorientierten Wildwuchs von Interventionen mit einem am systemischen Denken orientierten behutsamen Interventionskonzept zu begegnen. Der vorliegende Beitrag versucht, durch Vorstellen eines Analyserahmens und einer daraus abgeleiteten Interventionsstrategie einen Schritt in diese Richtung zu machen.

\section{Ein Analyserahmen zur Bestimmung von Ansatz- punkten für entwicklungspolitische Interventionen}

Entwicklung wird hier in einem relativ ergebnisoffenen und für alle Gesellschaften anwendbarem Sinn verstanden, als «Abfolge von gesellschaftlichen Problemlösungsprozessen basierend auf zunehmender Problemlösungsfähigkeit von Menschen und ihrer Institutionen» (vgl. RAUCH 1996). Dieser Entwicklungsbegriff setzt bei der Lösung von Problemen, also beim menschlichen Handeln an.

Betrachten wir die Einflussfaktoren auf Entwicklung, so gelangen wir zu der Feststellung, dass menschliches Handeln sich innerhalb bestimmter, sich wandelnder, durch Natur, Kultur, Gesellschaft, Politik und Ökonomie vorgegebener Handlungsspielräume abspielt. Mit anderen Worten: Das Handeln von Akteuren wird durch von diesen nicht unmittelbar beeinflussbaren Rahmenbedingungen begrenzt. Diese ökonomischen, politischinstitutionellen, sozio-kulturellen und ökologischen Rahmenbedingungen aber begrenzen nicht nur, sie schaffen und gestalten auch Handlungsspielräume 
und Handlungsanreize. Rechtssicherheit beispielsweise begrenzt Handlungsspielräume und schafft dadurch gleichzeitig Handlungsspielräume und -anreize. Da unterschiedliche Akteure unterschiedliche Einflusssphären und -möglichkeiten haben, sind sie mit unterschiedlichen Konstellationen von Rahmenbedingungen und Handlungsspielräumen konfrontiert. Entscheidungen des Direktoriums der Weltbank gestalten Handlungsspielräume von Finanzministern in sog. Entwicklungsländern. Agrarpolitische Entscheidungen von Regierungen setzen Rahmenbedingungen und gestalten Handlungsspielräume von Bäuerinnen und Bauern, Nahrungsmittelkonsumentinnen und -konsumenten. Rahmenbedingungen bzw. Handlungsspielräume und die Handlungsstrategie von Akteuren beeinflussen einander also wechselseitig.

Die Art dieses interdependenten Verhältnisses ist seit langem Gegenstand der Auseinandersetzung um gesellschaftswissenschaftliche Paradigmen (vgl. den Beitrag von TRÖGER in diesem Heft). Strukturtheoretische Ansätze nehmen die als Resultat von Handlungen entstandenen Strukturen zum zentralen Betrachtungsgegenstand und glauben vom Handeln einzelner Akteure in einem hohen Masse abstrahieren zu können, weil dieses sich nach bestimmten, durch die Strukturen bzw. Systemmechanismen weitgehend vorgegebenen Regeln abspielt. Zwar wird das Handeln der Individuen nicht notwendigerweise als strukturell determiniert angesehen, doch die Summe allen individuellen Handelns bestimmter Kategorien von Akteuren (z. B. der Arbeiter, der Unternehmer) wird als prognostizierbare Funktion von Strukturen gesehen. Die Vielfalt individueller Handlungsstrategien wird bestenfalls als interessant, nicht aber als erheblich für die Suche nach den Triebkräften und Mechanismen von Gesellschaften gesehen. Im Bereich der Entwicklungstheorien sind sowohl die modernisierungsals auch dependenztheoretischen Ansätze, aber auch die neueren Beiträge zu den Globalisierungsfolgen (vgl. Hein 2002) der Kategorie strukturtheoretischer Ansätze zuzuordnen.

Handlungs- bzw. akteurstheoretische Ansätze (vgl. hierzu den Beitrag von TRÖGER in diesem Heft) hingegen richten ihr Augenmerk in erster Linie auf die Analyse der Akteure und ihrer Handlungsstrategien. Für sie steht die strukturprägende, vor allem aber die strukturverändernde Kraft menschlichen Handelns im Vordergrund der Betrachtung (vgl. die Strukturationsthese von GidDENS 1997 und WerLen 1997). Die einzelnen Akteure werden als die entscheidenden Triebkräfte von Veränderungsprozessen gesehen.

Der hier vorgestellte Analyserahmen begreift sich als Versuch einer Synthese zwischen struktur- und handlungstheoretischen Ansätzen. Hier steht das wech- selseitige Verhältnis zwischen Struktur und Handeln, zwischen Rahmenbedingungen und den Handlungsstrategien der verschiedenen Akteure im Zentrum der Betrachtung. Hier wird nicht a priori eine Dominanz der Strukturen oder eine hohe strukturprägende Gestaltungsmacht von Akteuren paradigmatisch vorausgesetzt, sondern - ergebnisoffen - als Resultat historischer Konstellationen betrachtet und damit als Gegenstand konkreter, historisch-spezifischer Theorien mittlerer Reichweite. Dem liegt die Hypothese zugrunde, dass Handeln in manchen historischen Situationen (z.B. Feudalismus) hochgradig strukturbestimmt ist, während es z.B. in historischen Umbruchsituationen relativ große Freiheitsgrade für strukturveränderndes Handeln gibt (vgl. TRÖGER 2003). Diese Herangehensweise erfordert pragmatischen Theorien-Pluralismus, welcher weder auf strukturalistische Analysen verzichtet, wenn es darum geht, die Bewegungsmechanismen der globalen Kapitalmärkte im Hinblick auf die Ausgrenzung von Regionen und der dort lebenden Bevölkerungsgruppen zu analysieren, noch auf handlungstheoretische Betrachtungen, wenn es darum geht herauszufinden, wie verschiedene Bevölkerungsgruppen mit den Folgen einer solchen Ausgrenzung umgehen.

Entsprechend dem in Abb. 1 dargestellten Analyserahmen wird vorausgesetzt (vgl. RAUCH 1996):

(1) Es gibt Handlungsspielräume begrenzende bzw. gestaltende und Handlungsanreize schaffende, von den jeweiligen lokalen Akteuren nicht oder nur schwer beeinflussbare Rahmenbedingungen (vgl. Tab. 1). Dabei gilt es zu unterscheiden zwischen:

- ökonomischen Rahmenbedingungen, der Sphäre der Märkte, deren Triebkräfte und Systemmechanismen in Zeiten fortschreitender Globalisierung vor allem auf globaler Ebene zu suchen sind,

- politisch-institutionellen Rahmenbedingungen, der Sphäre von Macht und Regulierungen, welche - wenngleich mit abnehmendem Grad an Entscheidungsautonomie - primär von Regierungen auf nationaler Ebene gesetzt werden,

- sozio-kulturellen Rahmenbedingungen, der Sphäre der Normen, Werte und Verhaltensmuster, welche meist ethnisch, religiös oder milieumäßig sich definierenden Teilgruppen nationaler $\mathrm{Ge}$ sellschaften zuzuordnen sind (es kann sich hier um regionale Gemeinschaften, aber auch um international verbreitete Gemeinschaften mit gemeinsamer Identität handeln),

- ökologischen Rahmenbedingungen, der Sphäre der Naturgesetze, welche bestimmten lokalen, regionalen oder auch grenzüberschreitenden Naturräumen zuzuordnen sind. 


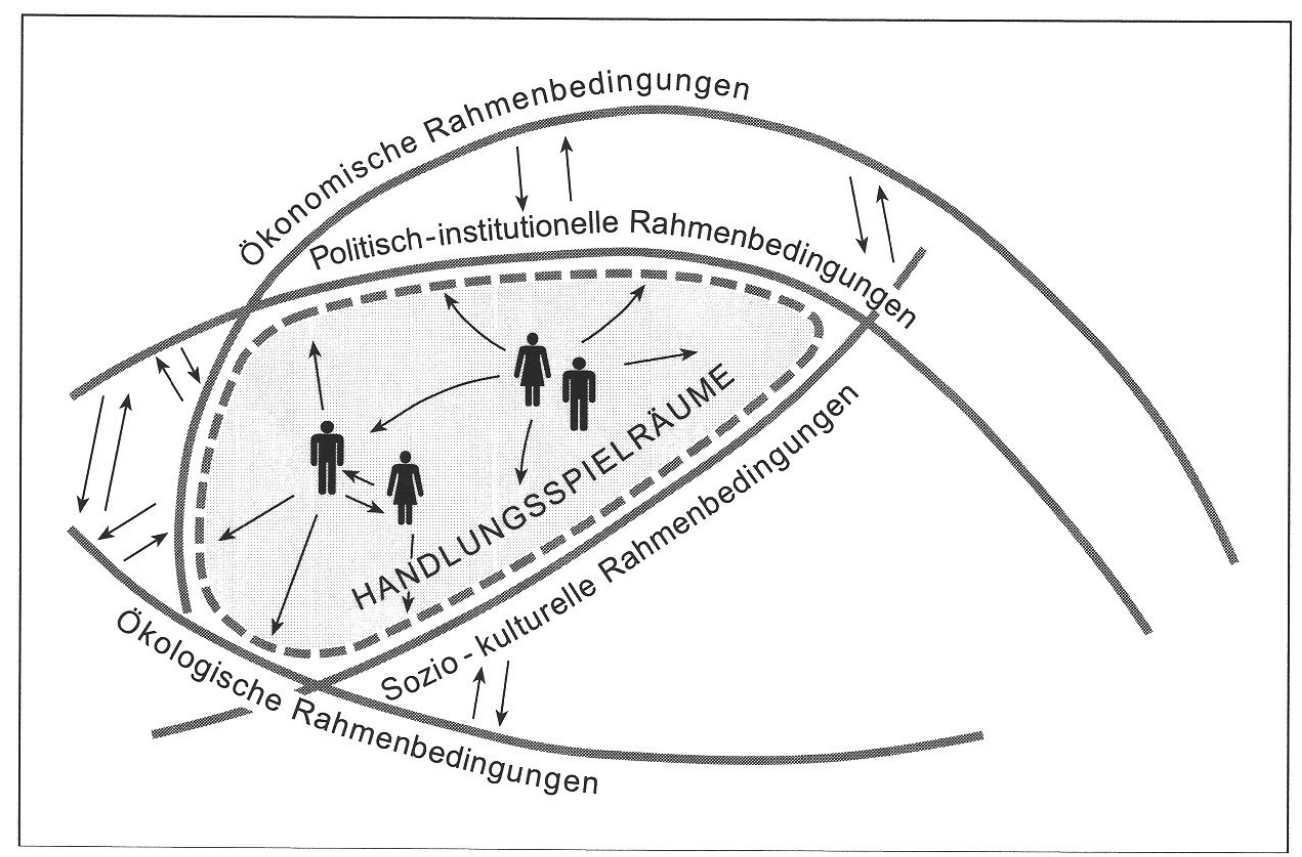

Abb. 1: Analyserahmen für armutsorientierte Interventionen

Analytical framework for poverty-related interventions

Cadre d'analyse pour des interventions orientées vers la pauvreté

Entwurf: T. RAUCH

(2) Diese Rahmenbedingungen beeinflussen einander wechselseitig und bilden für die lokalen Akteure (z.T. auch widersprüchliche) Handlungsvorgaben und -begrenzungen. Beispielsweise ist die fortschreitende Globalisierung von Märkten und Konsummustern mit einem Abbau politischinstitutioneller Gestaltungsmacht auf nationaler Ebene und mit einer Angleichung von Werten und Verhaltensmustern verbunden. Allerdings gibt es auch Beispiele dafür, dass das gemeinsame Handeln von Regierungen, z.B. bei der Umweltgesetzgebung, auf die globalen Märkte zurückwirkt oder dass kulturspezifische Konsummuster der Verbreitung von Weltmarktgütern gewisse Schranken setzen.

(3) Die Rahmenbedingungen lassen den lokalen Akteuren unterschiedlich große Handlungsspielräume, die sich im Extremfall auf Null (d.h. vollständige Determiniertheit) verengen können.

(4) Lokale Akteure konkurrieren innerhalb der für die Gemeinschaften verfügbaren Handlungsspielräume um begrenzte Möglichkeiten. Sie können aber auch zwecks verbesserter Nutzung oder Ausweitung von Handlungsspielräumen kooperieren. Als Resultat solcher Konkurrenz- bzw. Kooperationsbeziehungen ergeben sich unterschiedlich große individuelle Handlungsspielräume für verschiedene Akteure. Die Analyse darf also nicht von lokalen Gemeinschaften oder Haushalten als homogenen Akteursgruppen ausgehen. Es ist stets nach sozialen Gruppen sowie Geschlecht und Alter innerhalb einer Gemeinschaft zu differenzieren.

(5) Rahmenbedingungen schaffen nicht nur Handlungsmöglichkeiten, sondern auch Handlungsanreize bzw. -entmutigungen, welche die Handlungsstrategien nicht begrenzen, aber deren Attraktivität beeinflussen.

(6) Es kann nicht davon ausgegangen werden, dass lokale Akteure in der Lage sind, ihre Handlungsspielräume stets in bestmöglicher Weise zur Problemlösung bzw. Verbesserung ihrer Situation zu nutzen. Die Handlungsstrategien erzielen aufgrund begrenzter Informationen, begrenzter individueller Fähigkeiten, aufgrund von Wahrnehmungsmustern oder aufgrund von habituell vorgeprägten Reaktionsmustern (vgl. Beitrag von DöRFLER et al. in diesem Heft) nicht immer die erwünschte Wirkung oder es werden nicht alle vorhandenen Möglichkeiten wahrgenommen und genutzt. Dies spielt insbesondere bei sich schnell verändernden Rahmenbedingungen eine Rolle. 


\begin{tabular}{|c|c|c|c|c|c|}
\hline $\begin{array}{l}\text { Wirkungs- } \\
\text { dimension } \\
\text { Verursachende } \\
\text { Dimension }\end{array}$ & $\begin{array}{l}\text { Ökonomische } \\
\text { Dimension }\end{array}$ & $\begin{array}{l}\text { Politisch- } \\
\text { institutionelle } \\
\text { Dimension }\end{array}$ & $\begin{array}{l}\text { Sozio-kulturelle } \\
\text { Dimension }\end{array}$ & $\begin{array}{l}\text { Ökologische } \\
\text { Dimension }\end{array}$ & $\begin{array}{l}\text { Vorherrschende } \\
\text { Handlungsstrategien }\end{array}$ \\
\hline $\begin{array}{l}\text { Ökonomische } \\
\text { Dimension }\end{array}$ & $\begin{array}{l}\text { Vordringen von } \\
\text { Weltmarktproduktion } \\
\text { Reduzierte } \\
\text { Marktchancen } \\
\text { Neue Marktnischen }\end{array}$ & $\begin{array}{l}\text { Abbau von } \\
\text { Importschranken/ } \\
\text { Protektionismus }\end{array}$ & $\begin{array}{l}\text { Änderung der } \\
\text { Konsummuster } \\
\text { Neue Werte/ } \\
\text { Verhaltensanforderun- } \\
\text { gen der Märkte }\end{array}$ & $\begin{array}{l}\text { Tendenz zu Raubbau } \\
\text { zwecks } \\
\text { Devisenerwirtschaf- } \\
\text { tung } \\
\text { Treibhauseffekt }\end{array}$ & $\begin{array}{l}\text { Diversifizierung von } \\
\text { Überlebenssicherungs- } \\
\text { quellen } \\
\text { Überforderung }\end{array}$ \\
\hline $\begin{array}{l}\text { Politisch- } \\
\text { institutionelle } \\
\text { Dimension }\end{array}$ & $\begin{array}{l}\text { Verbesserte } \\
\text { Verwertungsbedingun- } \\
\text { gen }\end{array}$ & $\begin{array}{l}\text { Demokratisierung, } \\
\text { Liberalisierung, } \\
\text { Privatisierung } \\
\text { rent-seeking } \\
\text { Klientelismus }\end{array}$ & $\begin{array}{l}\text { Staatliches Bildungs- } \\
\text { system untergräbt } \\
\text { traditionelle } \\
\text { Machtstrukturen und } \\
\text { Werte }\end{array}$ & $\begin{array}{l}\text { Inadäquate staatliche } \\
\text { Kontrolle natürlicher } \\
\text { Ressourcen fördert } \\
\text { Raubbau }\end{array}$ & $\begin{array}{l}\text { nicht einheitliche } \\
\text { Handlungsstrategien }\end{array}$ \\
\hline $\begin{array}{l}\text { Sozio-kulturelle } \\
\text { Dimension }\end{array}$ & $\begin{array}{l}\text { Traditionelle } \\
\text { Konsummuster als } \\
\text { Schranke für } \\
\text { Weltmarktprodukte }\end{array}$ & $\begin{array}{l}\text { Patronageerwartung } \\
\text { stabilisiert } \\
\text { Klientelismus } \\
\text { Ethnizität erschwert } \\
\text { demokratische } \\
\text { Kontrolle }\end{array}$ & $\begin{array}{l}\text { Normenpluralismus } \\
\text { Normenvakuum } \\
\text { Moralische Krise }\end{array}$ & $\begin{array}{l}\text { Verfall der Normen } \\
\text { und Regeln zum } \\
\text { Schutz der Natur }\end{array}$ & $\begin{array}{l}\text { Verunsicherung } \\
\text { Opportunistisches Handeln } \\
\text { Kriminalität } \\
\text { Neue Religions- } \\
\text { gemeinschaften }\end{array}$ \\
\hline $\begin{array}{l}\text { Ökologische } \\
\text { Dimension }\end{array}$ & $\begin{array}{l}\text { Ökologische } \\
\text { Degradierung } \\
\text { verschlechtert } \\
\text { Verwertungsbedingun- } \\
\text { gen }\end{array}$ & $\begin{array}{l}\text { Natürliche } \\
\text { Ressourcen als } \\
\text { Rentenquelle } \\
\rightarrow \text { Stabilisierung der } \\
\text { Rohstoffabhängig- } \\
\text { keit }\end{array}$ & $\begin{array}{l}\text { Zunehmende } \\
\text { Nutzungskonkurrenz } \\
\text { untergräbt Normen der } \\
\text { gemeinschaftlichen } \\
\text { Erhaltung von Ressour- } \\
\text { cen }\end{array}$ & $\begin{array}{l}\text { Tendenz zur } \\
\text { Verknappung und zur } \\
\text { Degradierung }\end{array}$ & $\begin{array}{l}\text { Intensivierung } \\
\text { Abwanderung } \\
\text { Verschärfter Raubbau }\end{array}$ \\
\hline \begin{tabular}{|l|} 
Vorherrschende \\
Handlungsstrategie
\end{tabular} & (Minimaler Einfluss) & $\begin{array}{l}\text { Stabilisierung } \\
\text { klientelistischer } \\
\text { Strukturen } \\
\text { Häufiger Macht- } \\
\text { wechsel ohne Struk- } \\
\text { turveränderungen }\end{array}$ & $\begin{array}{l}\text { z.T. anhaltende Krise } \\
\text { z.T. Individualisierung } \\
\text { z.T. Re-Traditionalisie- } \\
\text { rung; neue Religions- } \\
\text { gemeinschaften }\end{array}$ & $\begin{array}{l}\text { z.T. Degradierung } \\
\text { z.T. Stabilisierung }\end{array}$ & $\begin{array}{l}\text { Vielfalt von o.g. Strategien; } \\
\text { oft widersprüchlich und } \\
\text { konfliktreich } \\
\text { Kampf mit Gewinnern und } \\
\text { Verlierern }\end{array}$ \\
\hline
\end{tabular}

Tab. 1: Wechselseitige Beziehungen zwischen den vier Dimensionen/Rahmenbedingungen Interdependent relations between the four dimensions/frameworks Rapports réciproques entre les quatre niveaux/conditions générales Entwurf:T. RAUCH

Das alte Wissen eignet sich dann nicht mehr für den Umgang mit den neuen Bedingungen. Als Konsequenz bleiben Handlungsspielräume ungenutzt; die tatsächlichen Handlungsräume sind vergleichsweise begrenzter, hinterlassen ein Vakuum.

(7) Andererseits beschränken sich Akteure nicht notwendigerweise auf die abgesteckten Handlungsspielräume, sondern versuchen mit ihren Strategien entweder deren Veränderung zu bewirken (bewusst oder als unbeabsichtigter Gesamteffekt der Strategien vieler Einzelner) oder aber sie missachten diese durch eine grenzüberschreitende Strategie (Missachtung von Gesetzen, Ignorierung von Normen, zerstörerischer Umgang mit den natürlichen Lebensgrundlagen). Auf dieses Phänomen der «Strukturation» geht TRöGER ausführlich ein (TRÖGER, in diesem Heft).

(8) Auch die unterbleibende Nutzung von Handlungsspielräumen kann zu deren Veränderung führen, indem sie andere Akteure dazu ermutigt, das so entstandene Vakuum zu füllen und damit die Rahmenbedingungen $\mathrm{zu}$ verändern. Ungenutzte natürliche Ressourcen können von externen Landnehmern in Besitz genommen werden. Ungenutzte Mitbestimmungsspielräume können zur Machtergreifung Einzelner und, daraus folgend, zur Abschaffung der Partizipationsrechte genutzt werden. Ungenutzte Marktnischen werden von einer externen Konkurrenz erobert.

Dieser modellhafte, ahistorische Analyserahmen gibt bereits implizit Hinweise darauf, dass es bei entwicklungspolitischen Interventionen grundsätzlich zwei verschiedene Ansatzpunkte gibt:

- die Beeinflussung der Rahmenbedingungen auf globaler, nationaler und regionaler Ebene (welche sich aus Sicht von entwicklungspolitischen Interventionsagenturen anders darstellen, als aus Sicht der lokalen Akteure) zwecks Erweiterung oder Umgestaltung der lokalen Handlungsspielräume,

- die Befähigung der lokalen Akteure zur besseren Nutzung und eventuell Erweiterung oder Restrukturierung ihrer Handlungsspielräume, d.h.Kapazitätsentwicklung und empowerment auf lokaler Ebene. 


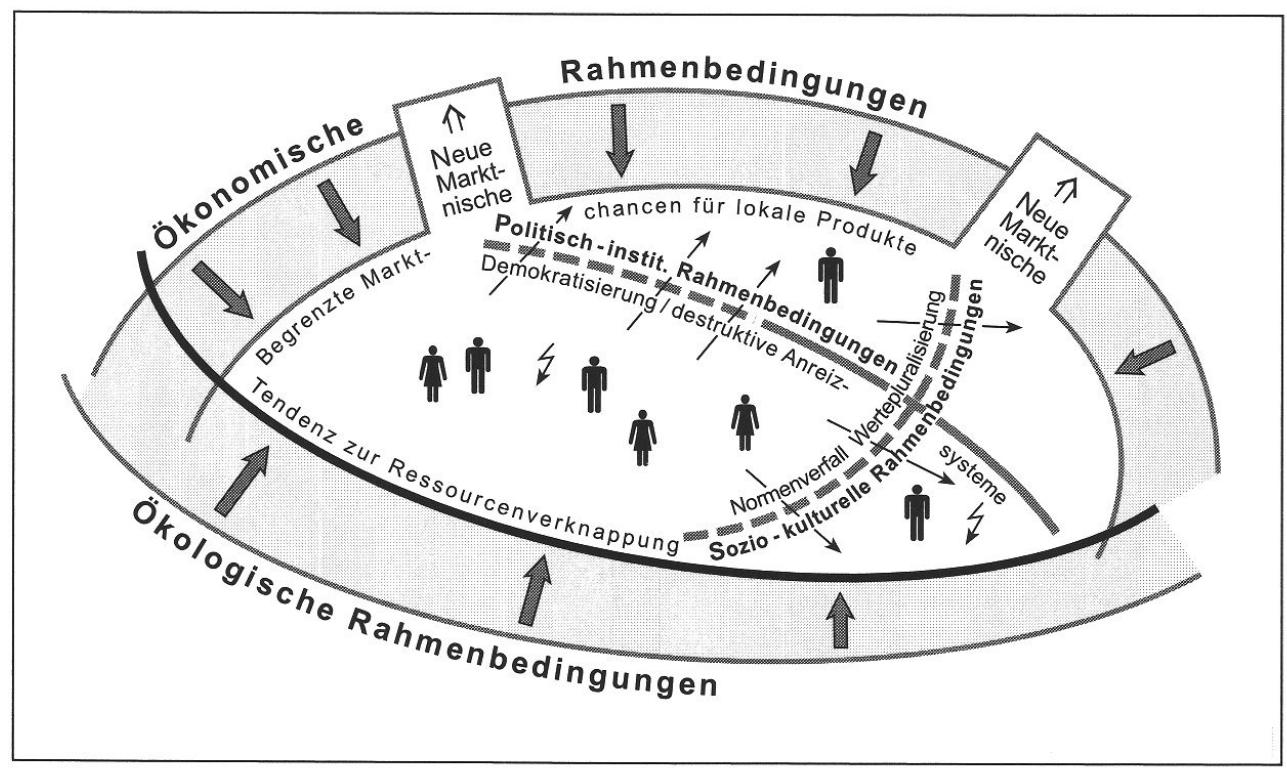

Abb. 2: Szenario einer Armutsregion unter demVorzeichen von Globalisierung und Ausgrenzung Poverty scenario in the context of globalisation and marginalisation

Scénario d'une région pauvre soumise à la globalisation et à la marginalisation

Entwurf: T. RAUCH

Struktur- bzw. systemtheoretisch beeinflusste Interventionsstrategien neigen eher zur Veränderung der Rahmenbedingungen, handlungstheoretisch geprägte Fachleute tendieren zur Befähigung lokaler Akteure. Bevor das im Untertitel dieses Beitrags angekündigte Vier Ebenen-Interventionsmodell dargestellt wird, soll hier zur Illustration ein Szenario von Rahmenbedingungen und Handlungsspielräumen entworfen werden, das der realen Problemkonstellation in vielen armutsgeprägten Regionen der Länder des sog. Südens nahe kommt. Dadurch soll auch der inhaltlich-substantielle Teil der vorgeschlagenen Interventionsstrategie plausibel gemacht werden.

\section{Ein typisches Szenario für eine von Massenarmut gekennzeichnete Region}

Aufgrund der gebotenen Kürze der Darstellung kann hier nur ein holzschnittartig verallgemeinertes Szenario skizziert werden, welches dennoch nicht zufällig Ähnlichkeiten mit vielen Fallstudien aus afrikanischen, asiatischen und lateinamerikanischen Regionen aufweist (vgl. Abb. 2).

- Die globalen ökonomischen Rahmenbedingungen tendieren in von den Globalisierungsprozessen ausgegrenzten Regionen dazu, durch verstärktes Vordringen von Importwaren auf lokale Märkte die Chancen zur Verbesserung der Einkommens- und Beschäftigungsmöglichkeiten eng $\mathrm{zu}$ begrenzen.
Der verschärften Binnenmarktkonkurrenz stehen zwar für manche Regionen und Produktionsländer neue Marktchancen auf externen Märkten gegenüber. Diese sind aber i.d.R. eng begrenzt und hart umkämpft. Angesichts der teilweisen, aber beschränkten Ausweitung wirtschaftlicher Handlungsspielräume gibt es in den meisten Regionen wenige Gewinner (z.B. Nutzer von Nischenmärkten) und viele Verlierer (vgl. Hein 1999 und Scholz 2000).

- Auf nationalstaatlicher Ebene schafft die mit Liberalisierung, Privatisierung, Demokratisierung und (z.T.) Dezentralisierung vollzogene Veränderung der (bislang meist sehr einengenden) politisch-institutionellen Rahmenbedingungen (per se) neue politische und wirtschaftliche Handlungsspielräume. Die durch die Marktliberalisierung vergrößerten ökonomischen Handlungsspielräume werden meist aber nur in den Bereichen Handel und lokales Dienstleistungsgewerbe von lokalen Akteuren genutzt. Ansonsten bleiben für lokale Eliten der Zugang zu Renteneinkommen über Positionen im Staatsapparat angesichts der ökonomischen Rahmenbedingungen weiterhin die attraktivste Einkommensquelle (vgl. ELSENHANS 1997), während die ressourcenarmen Akteure meist $\mathrm{zu}$ den Verlierern beim Zugang zu begrenzten wirtschaftlichen Möglichkeiten zählen. Die aus der Demokratisierung resultierenden vergrösserten politischen Handlungsspielräume bleiben aufgrund klientelistischer Abhängigkeiten meist unge- 
nutzt. In vielen, insbesondere ländlichen, Regionen hinterlässt der mit Privatisierungsbestrebungen verbundene Rückzug des Staates aus ökonomischen und sozialen Dienstleistungen ein Vakuum, da den privaten Akteuren entweder die Kapazitäten oder die Anreize fehlen, die Lücke zu schließen.

- Die sozio-kulturellen Rahmenbedingungen, d.h. die Normen und Werte, unterliegen einem durch die partielle Marktintegration (Monetarisierung) ausgelösten Wandel, welcher dadurch gekennzeichnet ist, dass die alten Werte und Regeln ihre Verbindlichkeit verloren haben, ohne dass sie aber durch ein neues Wertesystem abgelöst wurden. Es herrscht ein Wertepluralismus, ein normatives Vakuum, eine Beliebigkeit in der Berufung auf Regeln, kurz: eine moralische Krise. Die Starken innerhalb der lokalen Gemeinschaften nutzen diese Krise opportunistisch zu ihren Gunsten, den Schwächeren (die z.B. von der Einhaltung von Solidarnormen abhängig waren) bleibt die Verunsicherung (vgl. hierzu die Fallstudie aus Tansania von TRÖGER 2003 und den Beitrag von Tröger in diesem Heft). Hier wird der Wegfall von Rahmenbedingungen zum Problem.

- Die ökologischen Rahmenbedingungen sind häufig durch eine zur Verengung von Ressourcennutzungsspielräumen führende Degradierung natürlicher Ressourcen gekennzeichnet.

Der real in vielfältigen Variationen auftretende typische Problem-, sprich Armutsfall besteht also derzeit in vielen von der Globalisierungsdynamik ausgegrenzten Regionen darin, dass eine durch begrenzte Märkte und Ressourcenverknappung entstandene Einschränkung wirtschaftlicher Handlungsspielräume zusammentrifft mit dem Abbau von institutionellen und normativen Begrenzungen von Handlungsspielräumen. Das bedeutet neue individuelle Freiheiten bei insgesamt begrenzten Möglichkeiten. Diese werden tendenziell von den Starken zu Lasten der Schwachen genutzt. Hoffnungsvoll angestrebte neue Handlungsspielräume entpuppen sich oft als Vakuum (Abbau sozialer Dienste, z.B. im Gesundheits- und Erziehungswesen, in ländlichen Regionen) oder als Kampfarena.

Typische Handlungsstrategien in dieser Konstellation sind:

- Diversifizierung ökonomischer und sozialer Aktivitäten (auch in regionaler Hinsicht),

- Zunahme asozialer, unsolidarischer und krimineller Praktiken,

- Rückkehr zu alten religiösen Normen (Fundamentalismus) oder Hinwendung zu neuen Religionsgemeinschaften mit rigiden Normen,

- Verunsicherung und Hilflosigkeit mit verzweifelten Überlebensaktionen und Suche nach Hilfe.

Dort, wo die Möglichkeiten objektiv begrenzt sind, führen die zahlreichen Problemlösungsdiskurse meist nicht zu einem konstruktiven Ergebnis - es sei denn, sie werden von einer Hilfsorganisation gesteuert, bei welcher man am Ende des Diskurses einen Antrag stellen kann.

\section{Ein Vier Ebenen-Interventionsmodell}

Das Szenario konnte illustrieren, was sich auf abstrakter Ebene bereits als Konsequenz aus dem Analyserahmen anbot: Es ist notwendig, mit Interventionen bei Rahmenbedingungen und bei Handlungsstrategien von Armutsgruppen gleichzeitig anzusetzen, wenn die Entwicklung effektiv gefördert, wenn Armut vermindert werden soll. Zu den Gründen gehören:

- Die durch verbesserte Rahmenbedingungen geschaffenen neuen Handlungsspielräume können von lokalen Akteuren und insbesondere von den Armutsgruppen nicht oder nicht konstruktivi. S. von breitenwirksamen und nachhaltigen Problemlösungen genutzt werden. Das ist bedingt durch das Fortbestehen alter klientelistischer Abhängigkeiten auf lokaler Ebene, durch begrenzten individuellen Zugang zu Ressourcen, durch fehlende Erfahrungen im Umgang mit den neuen Bedingungen (z.B. mit Märkten, mit Mitbestimmungsrechten, mit Eigenverantwortung für die Wasserversorgung, mit Landverknappung) und durch mangelndes Wissen über entsprechende Problemlösungsmöglichkeiten.

- Befähigung und empowerment (d.h. Stärkung der Interessenvertretungsmacht) allein stoßen bei bestehenden Rahmenbedingungen, bei eng begrenzten Opportunitäten sehr schnell an objektive Grenzen. Zwar ist es möglich, im Sinne der Strukturationsthese, Menschen zu Handlungsstrategien zu befähigen, welche zu einer Veränderung von sozio-kulturellen, ökologischen und lokalen politischen Rahmenbedingungen beitragen, etwa indem klientelistische Abhängigkeitsstrukturen durch demokratische Interessenvertretungsstrukturen ersetzt werden (wozu es i.d.R. eines langfristigen Ansatzes bedarf). Diese Möglichkeiten beschränken sich im Allgemeinen auf lokale Rahmenbedingungen. Makro-Rahmenbedingungen auf nationaler oder gar globaler Ebene sind meist ausser Reichweite solcher auf lokale Akteure bezogener Strategien.

Aus diesen Überlegungen resultiert ein Vier EbenenInterventionsmodell (vgl. RAUCH 1996), welches von einander ergänzenden Interventionen auf den verschiedenen Ebenen ausgeht (vgl. Abb. 3):

(1) Auf globaler Ebene geht es im Sinne des Konzeptes der «Globalen Strukturpolitik» des deutschen Entwicklungshilfeministeriums (BMZ) unter anderem darum, 


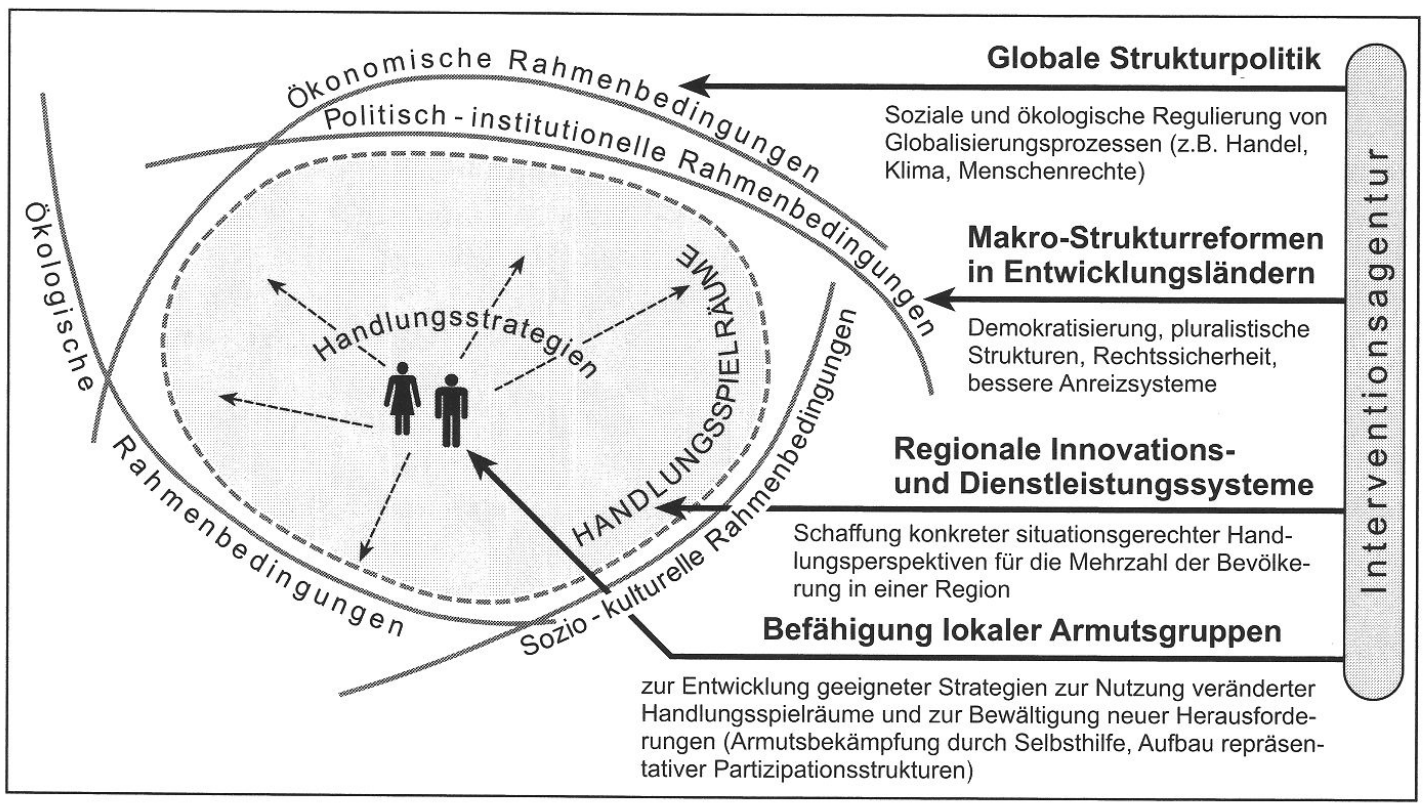

Abb. 3: Eine Vier Ebenen-Interventionsstrategie der Armutsminderung

A four-tiered strategy towards poverty reduction

Une stratégie des quatre niveaux en vue de la régression de la pauvreté

Entwurf: T. RAUCH

- die ökonomischen Globalisierungsprozesse durch Regulierungen im Rahmen der WTO sozial und ökologisch verträglich zu gestalten, was neben einem weiteren Abbau des Agrarprotektionismus der Industriestaaten gewisser Regulierungen des Welthandels und der Weltfinanzmärkte zum Schutze des Aufbaus einer umweltgerechten und beschäftigungswirksamen lokalen Wirtschaftsentwicklung bedarf,

- durch globale Umweltabkommen die Bedingungen für eine nachhaltige Ressourcennutzung und die Stabilisierung des Weltklimas zu verbessern,

- durch Menschenrechtspolitik individuelle Handlungsspielräume zu erweitern.

(2) Auf nationaler Ebene geht es darum, Makrostrukturreformen so zu gestalten, dass neue attraktive Handlungsfelder für Eliten in produktiven Bereichen entstehen, welche andere Optionen eröffnen, als die der Suche nach Rentenquellen durch die Erlangung von Positionen im Staatsapparat. Die Schaffung demokratischer Kontrollmechanismen muss ergänzt werden durch ein verändertes Anreizsystem. Das aber ist, wenn man die Erfahrungen der alten und der neuen Industrieländer zu Rate zieht, nicht durch die vollständige Öffnung der Grenzen, sondern nur durch selektiven, wohldosierten Protektionismus, also in Verbindung mit einer globalen Strukturpolitik möglich. Interventionen zugunsten demokratischer, pluralistischer und dezentraler Strukturen müssen dem tief verankerten Strukturmerkmal des Klientelismus Rechnung tragen, welches nicht ohne weiteres durch Verfassungsänderungen und Veränderungen des institutionellen Regelwerkes überwindbar ist, sondern nur durch den Abbau der ökonomischen Abhängigkeiten der Armen von den Zuwendungen der Reichen.

(3) Interventionen auf regionaler Ebene sind entscheidend, um reale neue Möglichkeiten für die Bevölkerung einer Region zu identifizieren und zugänglich zu machen. Es bedarf Bemühungen auf der regionalen, der Meso-Ebene, um Konzepte und Strategien $\mathrm{zu}$ entwickeln, welche gleichermaßen eine Antwort auf die Herausforderungen und Chancen der neu gestalteten, sich stetig verändernden Makro-Rahmenbedingungen sind, als auch den lokalen Bedingungen und Bedürfnissen innerhalb der Region Rechnung tragen. Regionale Konzepte und Strategien sind erforderlich, um für neue Handlungsspielräume breitenwirksame und nachhaltige Problemlösungsmöglichkeiten $\mathrm{zu}$ finden, um $\mathrm{zu}$ vermeiden, dass solche Handlungsspielräume nur einseitig von ökonomisch starken Akteuren genutzt werden (vgl. RAUCH et al. 2001). Regionale, sprich lokal 
angepasste und mit lokalen Nutzergruppen ausgehandelte Dienstleistungssysteme (z.B. Gesundheits-, Wasserversorgungs-, Kredit-, Vermarktungssysteme) sind nötig, um armen Bevölkerungsgruppen einen erschwinglichen und dauerhaften Zugang zu den erforderlichen sozialen und ökonomischen, staatlichen oder privaten Leistungsangeboten zu eröffnen. Träger für die Gestaltung (nicht unbedingt für die Bereitstellung!) solcher Innovations- und Dienstleistungssysteme sind i.d.R. regionale Gebietskörperschaften. Diese bedürfen meist externer Unterstützung für den Aufbau entsprechender Kapazitäten.

(4) Interventionen auf lokaler Ebene sind erforderlich, um arme Bevölkerungsgruppen dazu zu befähigen, Handlungsspielräume im Rahmen der geschaffenen Möglichkeiten zu nutzen. Dies erfordert einerseits Eigeninitiative bzw. lokale Selbstverantwortung, andererseits die Fähigkeit, über partizipative Strukturen eigene Bedürfnisse wirkungsvoll zu artikulieren. Dies kann gefördert werden durch Stimulierung und Moderation von lokalen Diskursen zur Frage des Umgangs mit dem Verfall verbindlicher Regeln und Normen sowie zur verbesserten Kooperation. Eine langfristige gesellschaftspolitsche Nebenwirkung solcher auf gemeinschaftliche lokale Problemlösungskapazitäten zielenden Interventionen kann die Ablösung klientelistischer Strukturen durch demokratische Interessenvertretungs- und Kontrollstrukturen sein, in denen die Führung nach Leistungskriterien gewählt wird.

Die Interventionen auf diesen vier Ebenen sind zumindest hinsichtlich ihrer grundsätzlichen Ausrichtung aufeinander abzustimmen. Handlungsspielräume können nicht per se erweitert werden, sondern müssen im Hinblick auf bestimmte Problemgruppen erweitert werden. Befähigung von Armutsgruppen kann nicht ohne weiteres erfolgen, sondern nur im Hinblick auf bestimmte Handlungsspielräume und -grenzen. Eine Feinabstimmung solcher Interventionen bezüglich Zeit und Raum mag zwar wünschenswert erscheinen, würde aber die Möglichkeiten der intervenierenden Organisationen übersteigen.

Insofern mag es hinreichend sein, wenn die Interventionen auf allen Ebenen einem gemeinsamen Rahmenkonzept mit gemeinsamen Grundsätzen folgen (wie z.B. Armutsorientierung, Nachhaltigkeit, Partizipation und Subsidiarität).

Die Beiträge von S. TrögER und C. MAYER in diesem Heft eignen sich als Beispiele für die Relevanz eines Mehr Ebenen-Interventionsansatzes. Am Beispiel Tansanias (Artikel von S. TRÖGER) wird deutlich, dass zwar einerseits die durch den Verfall einschlägiger Normen entstandenen Landnutzungskonflikte nicht ohne eine nationale Landrechtsreform lösbar erscheinen, dass aber andererseits eine derartige Veränderung der rechtlichen Rahmenbedingungen sich nur dann positiv auswirkt, wenn

- das Gesetzgebungsverfahren durch ortskundige regionale Akteure beeinflusst wird und so einen hinreichend flexiblen Rahmen für regions- und standortspezifische Umsetzungsstrategien und -programme bietet,

- lokale Gemeinschaften (und insbesondere die ärmeren, durchsetzungsschwächeren sozialen Gruppen) dabei unterstützt werden, die konkreten Landverteilungsfragen im Rahmen eines fairen, partizipativen und transparenten Verfahrens zu regeln.

Da es sich um neuartige Verfahren und Instrumente handelt, ist es in diesem Fall wahrscheinlich, dass ein Erfolg dieser Reform (im Sinne benachteiligter Gruppen) nur mit temporärer externer Unterstützung zu erwarten ist.

Das Beispiel der Öko-Siegel (Artikel von C. MAYER) lässt darauf schließen, dass derartige globale institutionelle Regulierungen nur dann auch zu situationsgerechten lokalen Ressourcennutzungsformen führen, wenn

- auf regionaler Ebene standort- und zielgruppengerechte, aber auch den ökologischen Grundsätzen des Siegels gerecht werdende Nutzungsformen identifiziert werden,

- auf lokaler Ebene Nutzergruppen an diesem Prozess beteiligt und bei der Umstellung unterstützt werden.

Ein anderes, prominentes Beispiel ist der Kampf gegen menschenverachtende Formen der Frauenbeschneidung. Auch hierzu bedurfte es einerseits internationaler Vereinbarungen auf der Weltfrauenkonferenz in Peking, nationaler Gesetzesänderungen, regionaler Prozesse der Einigung von relevanten staatlichen, zivilgesellschaftlichen Akteuren und insbesondere von traditionellen Würdenträgern auf eine gemeinsame Vorgehensweise, bevor einer einschlägigen Beratungsarbeit von Hilfsorganisationen auf lokaler Ebene Erfolg beschieden war (vgl. TeKÜLve 2001).

Bei manchen Lesern mögen sich bei so viel Interventionsbedarf auf allen Ebenen Bedenken einstellen. Wodurch sind diese Eingriffe legitimiert? Und wie können Einmischungen in derart sensible Systemzusammenhänge so gestaltet werden, dass am Ende nicht eine Verschärfung der Probleme steht?

Legitim sind Interventionen in soziale Systeme fremder Länder nur dann, wenn die letztendliche Entschei- 


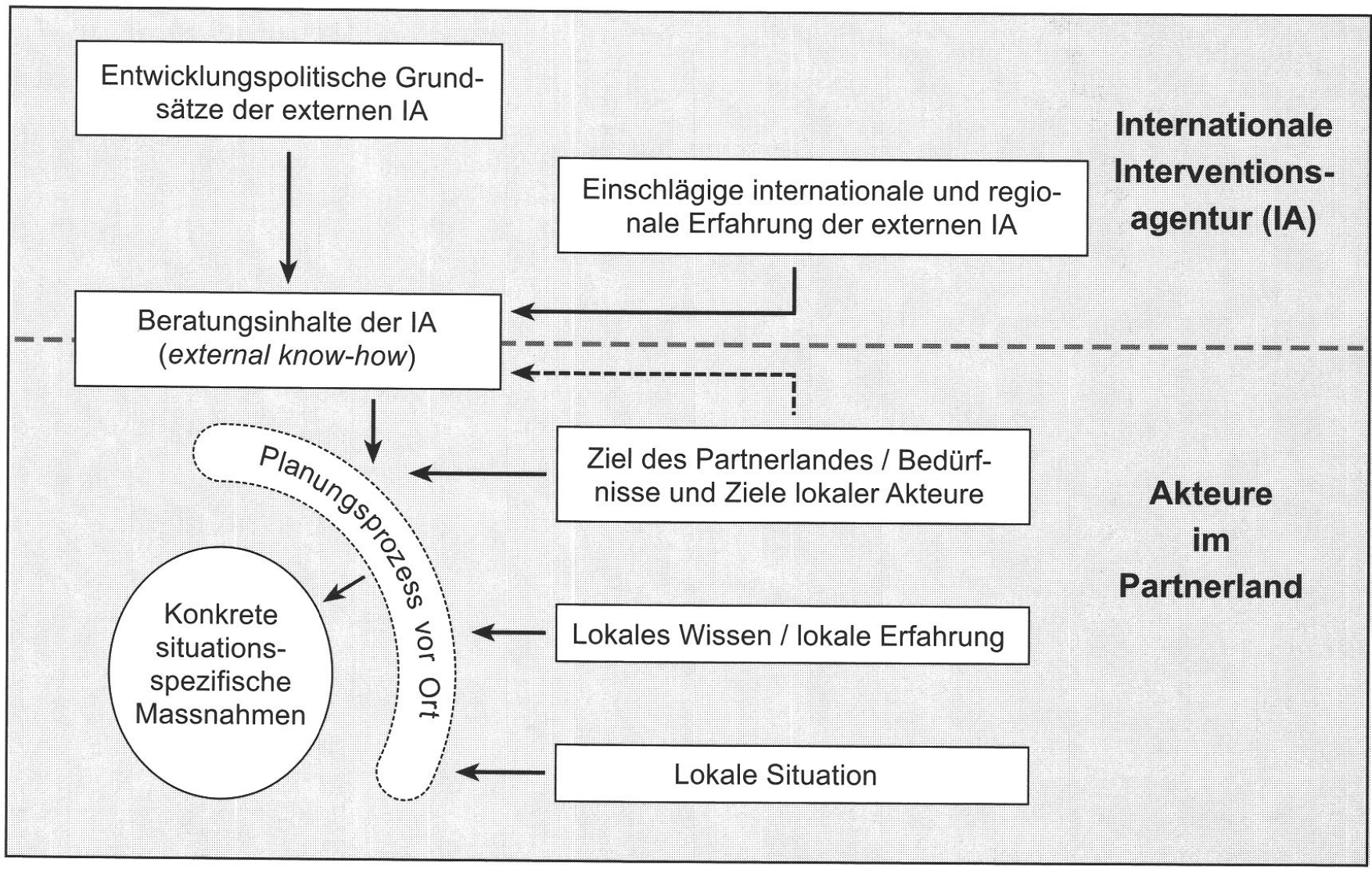

Abb. 4: Situationsgerechte Problemlösungen als Ergebnis der Verknüpfung von externen und lokalen Aspekten Appropriate problem solutions as a result of interlinking external and local factors

Des solutions adaptées aux problèmes posés, consécutives à l'articulation de facteurs externes et internes

Entwurf:T. RAUCH

dung darüber, was gemacht wird, bei den zuständigen Akteuren im Partnerland liegt. Von den Konsequenzen her fachlich und politisch vertretbar sind externe Interventionen in nur teilweise bekannte Systemzusammenhänge nur dann, wenn diese nicht als Patentrezept aufgedrängt werden, sondern im Rahmen eines gemeinsamen Planungs- und Entscheidungsprozesses als externes Wissen eingebracht werden, welches nur in Verknüpfung mit lokalem Wissen und der lokal spezifischen Problemlage zu situationsgerechten Problemlösungen führen kann (vgl. Abb. 4).

\section{Fazit}

Es gibt gute Gründe, warum die Basisarbeiter in den entwicklungspolitischen Institutionen und diejenigen, die auf der Ebene internationaler Konferenzen die Bedingungen für Armutsbekämpfung verbessern wollen, nicht weiter um knappe Mittel konkurrieren, sondern sich zusammentun und aufeinander abstimmen sollten. Angesichts der Dialektik zwischen Rahmenbedingungen und Handlungsoptionen für Problemlösungsanstrengungen armer Bevölkerungsgruppen wird weder der Ansatz der Makrostrukturreformer, noch jener der zielgruppennahen Befähiger es alleine schaffen. Basisarbeiter und -arbeiterinnen werden sich stets entweder auf kleinen, temporären Inseln relativer Glückseligkeit tummeln, auf denen sie die Bedingungen mit ihren Projektmitteln positiv gestalten können, oder sie werden über die widrigen, alle Anstrengungen zunichte machenden Rahmenbedingungen klagen. Makro-Interventionisten und -Interventionistinnen werden immer wieder betrübt feststellen müssen, dass ihre Reformen an der Basis nicht breitenwirksam sind.

Nur wenn beide Seiten ihre Interventionen aufeinander abstimmen, ist es wahrscheinlich, dass die von «oben» veränderten Handlungsspielräume «unten» auch von benachteiligten sozialen Gruppen problemlösend genutzt werden können. Der mittleren, regionalen Ebene fällt bei diesem wechselseitigen Abstimmungsprozess von politisch-ökonomischen Reformen und lokalen 
Handlungsstrategien eine besonders wichtige Rolle zu. Auf dieser Ebene gilt es aus abstrakten neuen Handlungsspielräumen innovative, konkrete Handlungsoptionen unter Berücksichtigung der jeweiligen regionalen Gegebenheiten zu konzipieren.

Das Zusammenwirken von Basisarbeitern und MakroInterventionisten ist aber auch aus einem anderen Grund wichtig: Nur durch Erfahrungsaustausch kann vermieden werden, dass auf der einen Seite Rahmenbedingungen von jenen gestaltet werden, die die reale Situation der davon betroffenen Menschen überhaupt nicht kennen, während auf der anderen Seite Zielgruppen in Unkenntnis der sich verändernden realen Möglichkeiten zu Handlungen motiviert und befähigt werden. Damit sind die Möglichkeiten, aber wohl auch die Grenzen der Entwicklungszusammenarbeit angedeutet.

\section{Literatur}

Dörfler, T., Graefe, O. \& D. Müller-Mahn (2003): Habitus und Feld - Anregungen für eine Neuorientierung der geographischen Entwicklungsforschung auf der Grundlage von Bourdieus «Theorie der Praxis».In: Geographica Helvetica 58/1:11-23.

Elsenhans, H. (1997): Staatskassen. - In: Schulz, M. (Hrsg.): Entwicklung. Die Perspektive der Entwicklungssoziologie: 161-186.

Giddens, A. (1997): Die Konstitution der Gesellschaft. Grundzüge einer Theorie der Strukturierung. - 3. Auflage, Frankfurt am Main, New York: Campus Verlag.

HeIn, W. (1999): Versunkenes Land? - Globalisierung, Archipelisierung und die Perspektiven marginalisierter Räume. - In: Nord-Süd aktuell, Nr. 3/1999: 403-417.

HeIn, W. (2002): Globalisierung und Regionalentwicklung. Neue theoretische Ansätze und die Chancen des Empowerment durch Global Governance. - In: NordSüd aktuell, Nr. 2/2002: 214-234.

MAYER, C. (2003): Können Öko-Siegel einen neuen Handlungsspielraum für kleinbäuerliche Kaffeeproduzenten schaffen? - Das Beispiel Costa Rica. - In: Geographica Helvetica 58/1: 56-65.

RAUCH, T. (1996): Ländliche Regionalentwicklung im Spannungsfeld zwischen Weltmarkt, Staatsmarkt und kleinbäuerlichen Strategien. - Saarbrücken: Verlag für Entwicklungspolitik Breitenbach.

Rauch, T., Bartels, M. \& A. Engel (2001): Regional Rural Developement. A regional response to rural poverty. - Wiesbaden, Berlin: Universum Verlagsanstalt.

Scholz, F. (2000): Globalisierung versus Fragmentierung. Eine regionalwissenschaftliche Herausforderung? - In: Nord-Süd aktuell, Nr. 2/2000: 255-271.

TeKÜLVE, M. (2001): Handlungsspielräume bestehen auf allen Ebenen. Zur Bedeutung von Armutsmin- derung und Gleichberechtigung in der Arbeit der GTZ. - Eschborn: Deutsche Gesellschaft für Technische Zusammenarbeit GmbH.

TRÖGER, S. (2003): Handeln zur Ernährungssicherung im Zeichen gesellschaftlichen Umbruchs. - Saarbrücken: Breitenbach Verlag (im Druck).

TRÖGER, S. (2003): Akteure in ihrer Lebensgestaltung (livelihood) zu Zeiten sozialer Transformation. Theoretische Überlegungen und ihre Anwendung auf das Beispiel von Landnutzungskonflikten in Tansania. In: Geographica Helvetica 58/1: 24-34.

WerLen, B. (1997): Gesellschaft, Handlung und Raum. Grundlagen einer handlungstheoretischen Sozialgeographie. - 3., überarbeitete Auflage, Stuttgart: Franz Steiner Verlag.

Wieczorek-Zeul, H. (2000): Aufgaben und Ziele Globaler Strukturpolitik im 21. Jahrhundert. - In: Jahrbuch Dritte Welt 2000. - München: Beck: 20-38.

\section{Zusammenfassung: Bessere Rahmenbedingungen allein beseitigen Armut nicht! Eine theoriegeleitete Vier Ebenen-Strategie für entwicklungspolitische Interventionen}

In der Auseinandersetzung um die sinnvollste Interventionsebene schlägt derzeit das Pendel des entwicklungspolitischen Mainstream zugunsten der globalen bzw. Makrostrukturpolitik aus. Anstelle der «Insellösungen» armutsorientierter Basisprojekte sollen die Armut verursachenden Rahmenbedingungen verändert werden. Der Artikel versucht zu begründen, warum diese plausibel klingende Strategie ebenso vom Fehlschlag bedroht ist wie all die isolierten Mikroebenen-Interventionen vergangener Jahrzehnte. Mit Hilfe eines Analyserahmens, welcher ein dialektisches Verhältnis von Rahmenbedingungen und Handlungsstrategien unterstellt, wird versucht, eine theoretische Grundlage für ein am Systemdenken orientiertes Interventionskonzept zu schaffen. Ergebnis dieser Vorgehensweise ist eine Vier Ebenen-Interventionsstrategie, im Rahmen welcher armutsorientierte Veränderungen globaler und nationaler Rahmenbedingungen, regionale Konzeptentwicklung und die Befähigung benachteiligter, armer Bevölkerungsgruppen zur Nutzung der neu entstehenden Handlungsspielräume aufeinander abgestimmt werden sollen.

\section{Summary: Improved Structural Conditions Alone Cannot Do Away With Poverty! A four-tiered theo- retical strategy for development intervention policy} In the debate about the appropriate level for poverty-related interventions, main-stream development policy currently favours macro-level or global level policy interventions. Instead of continuing to produce non-replicable «single aspect-solutions» through poverty-related micro-level projects, development cooperation agencies prefer to focus on changing the struc- 
tural conditions that cause povery. This article argues that a biased macro-level intervention strategy, no matter how plausible, is likely to fail just like most of the grass-root level efforts in previous decades. Based on an analytical framework which suggests an interdependent, dialectic relationship between structural conditions and personal action, a system-oriented concept for designing interventions is established. The outcome of this approach is a four-tiered intervention strategy which is to be used to coordinate povertyrelated changes of global and national frameworks and regional concept development, as well as to empower local level poverty groups so that they can make better use of the newly emerging fields of action.

\section{Résumé: La seule amélioration des conditions générales n'élimine pas la pauvreté! Une stratégie des quatre niveaux, fondée sur une réflexion théorique, en vue d'interventions politiques en faveur du déve- loppement}

La discussion relative au niveau le plus approprié d'intervention donne la faveur aux mesures prises dans le cadre d'une politique structurelle globale, respectivement d'une macrostructure politique. Les solutions insulaires fondées sur des projets de lutte contre la pauvreté doivent, pense-t-on, être remplacées par la prise en compte des conditions générales. La présente contribution tend à démontrer pourquoi cette stratégie, apparemment plausible, est autant vouée à l'échec que toutes les autres interventions entreprises au cours des décennies écoulées à un micro-niveau isolé. A l'aide d'un cadre d'analyse, qui sous-tend un rapport dialectique entre le cadre général et les stratégies d'intervention, nous créons la base théorique d'un concept orienté vers la réflexion systémique. Le résultat de cette démarche aboutit à une stratégie des quatre niveaux, susceptibles de permettre l'ajustement des changements destinés à lutter contre la pauvreté, au regard des conditions générales globales et nationales, du développement conceptuel régional, ainsi que de la possibilité donnée à des groupes pauvres de la population de bénéficier des nouveaux créneaux d'action.

\section{Didaktische Hinweise}

- Welche Probleme ergeben sich aus Entwicklungsbemühungen der vergangenen Jahrzente? Welche Forderungen stehen hinter dem Begriff Strukturanpassungsprogramm?

- In welcher Weise beeinflussen Rahmenbedingungen und Handlungsspielräume von Akteuren einander wechselseitig? Wie kann dieses Modell zur Erklärung von Entwicklungsprozessen herangezogen werden?

- Welche idealtypischen Veränderungstendenzen von Rahmenbedingungen und Handlungsspielräumen lassen sich derzeit in sog. Entwicklungsländern beobachten? Welche Probleme und welche Entwicklungschancen resultieren daraus?

- Welche sinnvollen Ansatzpunkte resultieren aus diesem Erklärungsmodell für die Gestaltung entwicklungspolitischer Interventionen? Warum ist eine Veränderung von Makro-Rahmenbedingungen nicht hinreichend für Armutsminderung? Worin liegt die spezifische Bedeutung regionaler Interventionen?

Prof. Dr. Theo Rauch, Zentrum für Entwicklungsländer-Forschung (ZELF), Geographisches Institut, Freie Universität Berlin, Malteserstrasse 74-100, D-12249 Berlin; Fax: 0049-228-5388856.

\section{Manuskripteingang/received/manuscrit entré le} 18.11.2002

Annahme zum Druck/accepted for publication/accepté pour l'impression: 4.4 .2003 